\title{
Reactive Oxygen Species in Status Epilepticus
}

Shekh-Ahmad $\mathrm{T}^{1,3^{*}}$, Kovac $\mathrm{S}^{2^{*}}$, Abramov $\mathrm{AY}^{4}$, Walker $\mathrm{MC}^{1}$

1. Department of Clinical and Experimental Epilepsy, Queen Square UCL Institute of Neurology, University College London, London, UK.

2. Department of Neurology, University of Muenster, Muenster, Germany.

3. School of Pharmacy and David R. Bloom Centre for Pharmacy, Faculty of Medicine, The Hebrew University of Jerusalem, Jerusalem, Israel.

4. Department of Molecular Neuroscience, UCL Institute of Neurology, University College London,

*These authors contributed equally

\begin{abstract}
There has been growing evidence for a critical role of oxidative stress in neurodegenerative disease, providing novel targets for disease modifying treatments. Although antioxidants have been suggested and tried in the treatment of epilepsy, it is only recently that the pivotal role of oxidative stress in the pathophysiology of status epilepticus has been recognised. Although conventionally thought to be generated by mitochondria, reactive oxygen species during status epilepticus and prolonged seizure are generated activity mainly by NADPH oxidase (stimulated by NMDA receptor activation). Excessive production of reactive oxygen species results in lipid peroxidation, DNA damage, enzyme inhibition and mitochondrial damage, culminating in neuronal death. Antioxidant therapy has been hampered by poor CNS penetration and rapid consumption by antioxidants. However, alternative approaches such as inhibiting NADPH oxidase or increasing endogenous antioxidant defences through activation of the transcription factor nuclear factor erythroid 2-related factor 2 (Nrf2) could avoid these problems. Small molecules that increase Nrf2 activation have proven to be not only effective neuroprotectants following status epilepticus, but also potently antiepileptogenic. There are a range of Nrf2 activators that are in clinical trial for other indications, suggesting that repurposing of these therapies could provide a rapid translation of these for the treatment of status epilepticus. This article is part of the Special Issue "Proceedings of the 7th London-Innsbruck Colloquium on Status Epilepticus and Acute Seizures.
\end{abstract}

Key words: status epilepticus, reactive oxygen species, Nrf2, NADPH oxidase, mitochondria, oxidative stress

Abbrevations: KEAP1, Kelch-like ECH associated protein 1; Nrf2, nuclear factor erythroid 2-related factor 2; ROS, reactive oxygen species; SE, status epilepticus; 


\section{Introduction}

There has been a strong evolutionary drive for organisms and cells to use atmospheric dioxygen for respiration to meet energy demands. However, oxygen also naturally occurs in more reactive forms (such as singlet oxygen or $\mathrm{H}_{2} \mathrm{O}_{2}$ ) or as free radicals (atom or groups of atoms with an unpaired electron which act as electron acceptors) such as superoxide anion $\left(\mathrm{O}_{2} \cdot-\right)$, hydroxyl radical $(\mathrm{OH})$; these are termed reactive oxygen species (ROS)[1]. ROS can be produced non-enzymatically by UV irradiation or enzymatically in the cells. ROS production varies greatly in different tissue depending on the availability of oxygen and expression of ROS-producing enzymes.

Compared to other organs, the brain is one of the greatest ROS producers because of its $\sim 10$-fold higher oxygen consumption and high metabolic rate compared to that of other tissues in the body[2]. The major ROS producers in the brain comprise NADPH oxidase (predominantly in two isoforms NOX2 and NOX4), mitochondria (electron transport chain, enzymes of TCA and monoamine oxidases A and $B,[3]$ ), xanthine oxidase and lipoxygenase. Brain ROS generation occurs even under resting conditions and greatly increases during brain activity, so that an effective antioxidant system is necessary to protect cells against oxidative stress. The major endogenous antioxidants in the brain, glutathione and a-tocopherol (vitamin E) in lipids[4,5], play important protective roles along with antioxidant enzymes[2]. The dependence of the level of ROS production on cellular activity and the short lifetime of superoxide and its consequent limited diffusion make ROS ideal signalling molecules, necessary for physiological function[3]. In the brain, a plethora of processes have been identified that are regulated by moderate amounts of ROS. For example, nicotinamide adenine dinucleotide phosphate-oxidase (NADPH oxidase)-derived ROS are instrumental in long term potentiation (LTP), a process required for memory formation[6].

However, changes of the redox balance in the brain through an increase in ROS production or a decrease in the level of antioxidants can lead to oxidative damage, cellular dysfunction and cell death. Excess ROS lead to lipid peroxidation and direct protein damage, which alters membrane and enzyme function, respectively, and subsequently destroys the cell[7]. The brain with its high content of polyunsaturated fatty acids is particularly prone to ROS-induced damage. Many neurological disorders are strongly associated with oxidative stress and excessive ROS production. Although energy deprivation plays a central role in ischaemia, significant reperfusion damage occurs through increased ROS production, particularly through NADPH oxidase activation during reoxygenation[8]. There is increasing evidence for the involvement of oxidative stress in Alzheimer's disease observed both in post-mortem tissue of Alzheimer's disease patients[9] and also in cellular systems[10]. In Parkinson's disease, all the toxic proteins that generate the pathology have been shown to activate ROS production, and most of proteins, encoded by Parkinson's associated genes, are involved in mitochondrial or redox homeostasis[2]. Moreover, aggregated protein $\alpha$ synuclein, which is involved in both sporadic and familial form of Parkinson's disease, is able to produce superoxide in combination with heavy metal ions, so triggering neuronal cell loss[11]. Mitochondrial ROS 
overproduction is also evident in many neurodegenerative diseases including Huntington's disease, amyotrophic lateral sclerosis, progressive supranuclear gaze palsy, Friedreich's ataxia, and frontotemporal dementia[12-15]. Although antioxidant therapy has been proven to be effective for a number of neurodegenerative disorders in experiments on a cellular level, most of the clinical trials have failed to demonstrate neuroprotection or efficacy in patients. This failure to translate the positive effects of antioxidants in experimental systems is usually attributed to the difficulties in antioxidant delivery to cells in the brain, or to the chemical instability of the antioxidant.

\section{ROS as a pathogenic mechanism in status epilepticus and excitotoxicity}

Pioneering studies in baboons by Meldrum and colleagues in the 1970's demonstrated that seizure activity, independent of any physiological compromise, can cause neuronal damage[16,17]. Further studies led to the idea that this damage was mediated through excitotoxicity from the excessive release of glutamate. As far back as 1957, glutamate was recognised as a potent neurotoxin[18]. Experiments on glutamate and its analogues demonstrated: that there is a correlation between their excitatory and toxic actions; that specific antagonists of their excitatory activity protect against their neurotoxicity, and that they are toxic to dendrites and cell bodies (dendrosomatotoxic) and axon-sparing. These observations led to the hypothesis that the toxic action was mediated through an action of glutamate at dendrosomatic receptors - the excitotoxic hypothesis[19]. Subsequent evidence indicated that ROS play a role in this excitotoxic damage[20], and later, ROS production was shown to be necessary for excitotoxic neuronal injury[21].

Oxidative stress during and after seizures was later shown to be instrumental in immediate and longer-term excitotoxic neuronal death[21,22], and the reduction of aconitase and glutathione shortly following status epilepticus strongly support this hypothesis[23]. Excessive ROS production (especially in combination with Nitric Oxide to form peroxynitrite, a reactive nitrogen species) leads to lipid peroxidation, inactivation of enzymes, mitochondrial permeability transition pore opening and DNA damage[24-26] (Fig. 1). Moreover, excessive ROS production can inhibit mitochondrial complex 1 activity, decrease mitochondrial membrane potential and inhibit ATP production[27-29].

Human data have largely confirmed the animal studies. Oxidative stress markers are increased in the hippocampus of humans who died following status epilepticus or with chronic pharmacoresistant epilepsy[30]. A further recent study has indicated that patients with status epilepticus have reduced antioxidants and increased reactive oxygen and nitrogen species. Furthermore, the study also showed that catalase glutathione and total antioxidant capacity were lower and malondialdehyde and nitric oxide levels were significantly higher in the status epilepticus patients with comorbidity[31]. Moreover, in progressive myoclonic epilepsy, there is considerable evidence that ROS production is a key component of the 
pathophysiology[32]. Thus, animal and human data confirm that oxidative stress and excessive ROS production are notable features in status epilepticus.

\section{Source of ROS in epilepsy}

Mitochondria, the key organelles in ATP production and metabolism, have been assumed to be a major source of ROS production during seizures. Complex I and III are the primary sites of ROS production in mitochondria through "electron leak" from the electron transport chain[33]; indeed, it has been proposed that complex III is the main contributor to superoxide production during seizure activity[34]. Mitochondrial ROS production is critically dependent upon mitochondrial function and a hyperpolarised mitochondrial membrane potential[35]. Prolonged seizure activity, however, leads to depolarization of the mitochondrial membrane potential and mitochondrial failure[36]. Therefore, although mitochondria could serve as a significant source of ROS during brief seizures, it is difficult to see how they can contribute significantly during status epilepticus. Using robust protocols for real-time in vitro and ex vivo monitoring of ROS production during hyperexcitability, we were able to establish that mitochondria are not the main source of ROS during prolonged seizure activity[29,37]. Mitochondria are, however, likely a prominent target of ROS induced damage in seizure activity and permeability transition pore opening which is strongly promoted by ROS has been shown to lead to cell death during prolonged seizure like activity[36].

In the recent years, there has been growing evidence for a role of NADPH oxidases in generating ROS in the brain. NADPH oxidases are enzymes specialized in ROS production and their initial discovery was through the study of the respiratory burst in phagocytes and granulocytes[38]. We have identified NADPH oxidase as an important source of hyperexcitability-induced ROS production and consequent neuronal damage[29] (Fig. 1). During electrically-induced status epilepticus prominent ROS production and subsequent neuronal cell death in hippocampal subfields can be observed. This was suppressed by pharmacological NADPH oxidase inhibition[37].

NADPH oxidases exist in different isoforms which have been shown to exert different functions such as cellular signalling or regulation of cell growth[39]. Moreover, within the brain, these isoforms are differentially expressed in different cells. In mammals, seven NADPH isoforms have been found, including NADPH oxidase (NOX)1-5 and two dual oxidases (DUOX1 and DUOX2)[39,40]. NOX1,2 and 4 are expressed in the brain[40]. Several studies have looked into the role of NADPH oxidases in epilepsy (for a summary see table in [7]). It emerges that NOX2 plays a prominent role, which has been confirmed with more specific pharmacological antagonists[7]. NOX2 has been shown to be activated via NMDA receptor activation[41], a receptor which has a prominent role in seizure and status epilepticus-induced neuronal death[42]. 
NADPH oxidase may also contribute to the pathophysiology of status epilepticus in other ways. Vasogenic oedema formation during status epilepticus has also been linked to endothelin $B$ (ETB) receptor-mediated astrocytic ROS production through NADPH oxidase activation[43]. NADPH oxidase has also been identified as a pharmacological target for increased seizure susceptibility after systemic inflammation[44] and NADPH oxidase inhibition with apocynin has been shown to lead to ameliorate seizure-induced reduction of adult hippocampal neurogenesis[45]. However, the precise roles of different NOX isoforms subtypes in hyperexcitability, seizures and epilepsy are still unclear and await further studies using genetic ablation or isoform specific inhibitors.

Although NADPH oxidase is an important mediator of ROS induced cell damage in seizures and epilepsy, several other ROS sources exist in the cell such as Xanthine oxidase, monoaminoxidase and cyclooxygenase and lipoxygenase; these can also contribute to seizure-induced ROS [29,46,47]. During status epilepticus the high metabolic demand results in excessive breakdown of ATP which enhances xanthine oxidase activity, and therefore contributes to a significant proportion of ROS production[29].

Establishing the sources of ROS at different time points and under different conditions in seizures, status epilepticus and epileptogenesis could reveal new important treatment targets. It is likely that different sources of ROS production are prominent at different phases of the disease - a phenomenon which has already been observed in ischemia reperfusion injury in the brain[8].

\section{Neuroprotective and antiepileptogenic role of ROS inhibition following status epilepticus}

The use of antioxidants for the treatment of epilepsy has a long history, with successful therapies being described as far back the 1970s [48]. The most extensively studied antioxidant therapy in epilepsy is $\alpha$ tocopherol or vitamin E. Animal studies have confirmed its antioxidant efficacy and indicated antiepileptic effects. It was shown that pretreatment of rats with combined $\alpha$-tocopherol and selenium prevented the development of iron-induced epileptiform activity in $72 \%$ of animals compared with $6 \%$ of untreated controls receiving an intracortical injection of aqueous iron[49]. However, an extensive study of $\alpha$-tocopherol in four animal models of seizures and epileptogenesis confirmed its efficacy in the ferrous chloride model but not in the pentylenetetrazole threshold model, the maximal electroshock model and the kindling model of epileptogenesis[50]. Furthermore, $\alpha$-tocopherol demonstrated negligible effects in preventing seizures and oxidative injury in animal models of limbic epilepsy, such as amygdala-kindled seizures and electricallyinduced SE[51].

Despite these findings, $\alpha$-tocopherol administration following kainic acid induced SE, significantly reduced astrocytosis and microglia activation, emphasizing its anti-inflammatory and neuroprotective potential[52]. This was confirmed in a recent study, which demonstrated that long-term treatment with $60 \mathrm{IU} / \mathrm{kg} / \mathrm{day}$ of vitamin $E$ prevented oxidative damage in the hippocampus and increased hilar parvalbumin expression in rats with epilepsy without a reduction in seizure frequency[53]. 
Numerous animal studies have demonstrated that the use of other antioxidants such as vitamin $\mathrm{C}, \mathrm{N}$-acetylcysteine, coenzyme Q10, and various plant extracts or flavonoids for the treatment of SE, reduce lipid oxidation and restore the activities of different antioxidant enzymes such as, superoxide dismutase, catalase, glutathione peroxidise and the levels of glutathione in the rat hippocampus, striatum or cortex[54-59]. Some antioxidants have shown particular promise including resveratrol, a naturally occurring antioxidant found in red grapes. Resveratrol given both before and after status epilepticus has been shown to decrease hippocampal neuronal cell death, mossy fiber sprouting and abnormal neurogenesis[60,61]. Similarly, melatonin, a naturally occurring hormone that is a potent antioxidant and free radical scavenger attenuates seizure activity and neuronal damage in the hippocampus and piriform cortex following kainic acid-induced SE[62], and AEOL10150, a metalloporphyrin catalytic antioxidant, resulted in reduced mortality, neuronal death and inflammation compared to control animals[63]. Antioxidant strategies, therefore, show considerable promise in neuroprotection in SE. However, administration of exogenous antioxidants provides specific challenges, and, as a consequence, the results from many studies are mixed. The antioxidant has to cross the blood brain barrier, and antioxidants get rapidly oxidized and so "consumed" through ROS scavenging, resulting in a short action. In addition, the translational potential of many of the studies is low as the antioxidant was given prior to the status epilepticus.

In view of this, we have investigated, other, more enduring, antioxidant strategies including decreasing ROS production by targeting NAPH oxidase[37] and increasing endogenous antioxidant defences through activation of nuclear factor erythroid 2-related factor 2 (Nrf2)[30,64]. Nrf2 is a transcription factor that is negatively regulated by Kelch-like ECH associated protein 1 (KEAP 1)[65] (Fig. 2). Nrf2 target proteins include not only antioxidant enzymes, but also enzymes involved in anti-inflammation, detoxification and metabolism[66]. Electrophiles and oxidants inactivate KEAP1 by chemically modifying critical cysteine sensors within the protein[67], leading to Nrf2 accumulation and increased transcription of Nrf2 target genes that encode antioxidants [68]. The long half-lives and the diverse nature of the upregulated proteins ensure long-lasting protection against multiple oxidants, offering protection against many pathologies[69]. In addition, Nrf2 activation results in an improvement of mitochondrial function, so increasing ATP production, which also can be neuroprotective[70]. Nrf2 increases after status epilepticus[71], probably as a response to increased ROS production. Genetically-induced overexpression of Nrf2 neuroprotects, reduces inflammation and decreases subsequent seizure frequency following status epilepticus[71].

Nrf2 can be activated by sulforaphane, a naturally occurring substance obtained from cruciferous vegetables such as broccoli. Sulforaphane in combination with the antioxidant $\mathrm{N}$-acetylcysteine given after status epilepticus decreased neuronal damage, subsequent seizures and cognitive deficits[30]. Sulforaphane is hampered by poor penetration of the CNS, so we tested a CNS penetrant, potent and specific inhibitor of KEAP1, RTA-408. RTA-408 given for 24 hours following status epilepticus offered almost complete 
neuroprotection in the hippocampus and reduced the occurrence of subsequent spontaneous seizures by $\sim 94 \%[64]$ (Fig. 3).

\section{Conclusion}

It is now well-established that status epilepticus results in an increase in ROS, which reach toxic levels resulting in neuronal death. Targeting oxidative stress following status epilepticus, using a variety of strategies, has been shown not only to neuroprotect, but also to prevent or ameliorate other consequences including cognitive decline and the occurrence of late, spontaneous seizures (epileptogenesis). There are a growing number of trials of potent and effective antioxidant strategies in other neurological disease and in other branches of medicine (in particular oncology) which could easily be repurposed for use in status epilepticus. Amongst the various strategies, activation of Nrf2, either alone or in combination with other antioxidant strategies, seems to hold the most promise.

\section{Funding:}

This work was supported by Epilepsy Research UK, and the European Union's Seventh Framework Programme (FP7/2007-2013) under grant agreement n602102 (EPITARGET). This work was undertaken at UCLH/UCL which receives a proportion of funding from the Department of Health's NIHR Biomedical Research Centres funding scheme.

\section{References}

[1] Apel K, Hirt H. REACTIVE OXYGEN SPECIES: Metabolism, Oxidative Stress, and Signal Transduction. Annual Review of Plant Biology 2004;55:373-99. doi:10.1146/annurev.arplant.55.031903.141701.

[2] Gandhi S, Abramov AY. Mechanism of Oxidative Stress in Neurodegeneration. Oxid Med Cell Longev 2012;2012. doi:10.1155/2012/428010.

[3] Angelova PR, Abramov AY. Functional role of mitochondrial reactive oxygen species in physiology. Free Radic Biol Med 2016;100:81-5. doi:10.1016/j.freeradbiomed.2016.06.005.

[4] Dringen R, Hirrlinger J. Glutathione pathways in the brain. Biol Chem 2003;384:505-16. doi:10.1515/BC.2003.059.

[5] Lee P, Ulatowski LM. Vitamin E: Mechanism of transport and regulation in the CNS. IUBMB Life 2019;71:424-9. doi:10.1002/iub.1993.

[6] De Pasquale R, Beckhauser TF, Hernandes MS, Giorgetti Britto LR. LTP and LTD in the visual cortex require the activation of NOX2. J Neurosci 2014;34:12778-87. doi:10.1523/JNEUROSCI.1414-14.2014.

[7] Kovac S, Dinkova Kostova AT, Herrmann AM, Melzer N, Meuth SG, Gorji A. Metabolic and Homeostatic Changes in Seizures and Acquired Epilepsy-Mitochondria, Calcium Dynamics and Reactive Oxygen Species. Int J Mol Sci 2017;18. doi:10.3390/ijms18091935.

[8] Abramov AY, Scorziello A, Duchen MR. Three distinct mechanisms generate oxygen free radicals in neurons and contribute to cell death during anoxia and reoxygenation. J Neurosci 2007;27:1129-38. doi:10.1523/JNEUROSCI.4468-06.2007.

[9] Lovell MA, Ehmann WD, Butler SM, Markesbery WR. Elevated thiobarbituric acid-reactive substances and antioxidant enzyme activity in the brain in Alzheimer's disease. Neurology 1995;45:1594-601. doi:10.1212/wnl.45.8.1594.

[10] Canevari L, Abramov AY, Duchen MR. Toxicity of amyloid beta peptide: tales of calcium, mitochondria, and oxidative stress. Neurochem Res 2004;29:637-50.

[11] Deas E, Cremades N, Angelova PR, Ludtmann MHR, Yao Z, Chen S, et al. Alpha-Synuclein Oligomers Interact with Metal lons to Induce Oxidative Stress and Neuronal Death in Parkinson's Disease. Antioxid Redox Signal 2016;24:376-91. doi:10.1089/ars.2015.6343. 
[12] Angelova PR, Barilani M, Lovejoy C, Dossena M, Viganò $M$, Seresini $A$, et al. Mitochondrial dysfunction in Parkinsonian mesenchymal stem cells impairs differentiation. Redox Biol 2018;14:474-84. doi:10.1016/j.redox.2017.10.016.

[13] Abeti R, Parkinson MH, Hargreaves IP, Angelova PR, Sandi C, Pook MA, et al. "Mitochondrial energy imbalance and lipid peroxidation cause cell death in Friedreich's ataxia." Cell Death Dis 2016;7:e2237. doi:10.1038/cddis.2016.111.

[14] Esteras N, Rohrer JD, Hardy J, Wray S, Abramov AY. Mitochondrial hyperpolarization in iPSC-derived neurons from patients of FTDP-17 with 10+16 MAPT mutation leads to oxidative stress and neurodegeneration. Redox Biol 2017;12:410-22. doi:10.1016/j.redox.2017.03.008.

[15] Ayala-Peña S. Role of oxidative DNA damage in mitochondrial dysfunction and Huntington's disease pathogenesis. Free Radic Biol Med 2013;62:102-10. doi:10.1016/j.freeradbiomed.2013.04.017.

[16] Meldrum BS, Brierley JB. Prolonged epileptic seizures in primates. Ischemic cell change and its relation to ictal physiological events. Arch Neurol 1973;28:10-7.

[17] Meldrum BS, Horton RW. Physiology of status epilepticus in primates. Arch Neurol 1973;28:1-9.

[18] Lucas DR, Newhouse JP. The Toxic Effect of Sodium L-Glutamate on the Inner Layers of the Retina. AMA Arch Ophthalmol 1957;58:193-201. doi:10.1001/archopht.1957.00940010205006.

[19] Olney JW, Collins RC, Sloviter RS. Excitotoxic mechanisms of epileptic brain damage. Adv Neurol 1986;44:85777.

[20] Coyle JT, Puttfarcken P. Oxidative stress, glutamate, and neurodegenerative disorders. Science 1993;262:689_ 95.

[21] Patel M, Day BJ, Crapo JD, Fridovich I, McNamara JO. Requirement for Superoxide in Excitotoxic Cell Death. Neuron 1996;16:345-55. doi:10.1016/S0896-6273(00)80052-5.

[22] Liang LP, Ho YS, Patel M. Mitochondrial superoxide production in kainate-induced hippocampal damage. Neuroscience 2000;101:563-70. doi:10.1016/S0306-4522(00)00397-3.

[23] Cock HR, Tong X, Hargreaves IP, Heales SJR, Clark JB, Patsalos PN, et al. Mitochondrial dysfunction associated with neuronal death following status epilepticus in rat. Epilepsy Res 2002;48:157-68.

[24] Szabó C, Ischiropoulos H, Radi R. Peroxynitrite: Biochemistry, pathophysiology and development of therapeutics. Nature Reviews Drug Discovery 2007;6:662-80. doi:10.1038/nrd2222.

[25] Moncada S, Bolaños JP. Nitric oxide, cell bioenergetics and neurodegeneration. Journal of Neurochemistry 2006;97:1676-89. doi:10.1111/j.1471-4159.2006.03988.x.

[26] Camello-Almaraz C, Gomez-Pinilla PJ, Pozo MJ, Camello PJ. Mitochondrial reactive oxygen species and Ca2+ signaling. American Journal of Physiology - Cell Physiology 2006;291:C1082-8. doi:10.1152/ajpcell.00217.2006.

[27] Ryan K, Backos DS, Reigan P, Patel M. Post-translational oxidative modification and inactivation of mitochondrial complex I in epileptogenesis. Journal of Neuroscience 2012;32:11250-8. doi:10.1523/JNEUROSCI.090712.2012.

[28] Rowley S, Liang L-P, Fulton R, Shimizu T, Day B, Patel M. Mitochondrial respiration deficits driven by reactive oxygen species in experimental temporal lobe epilepsy. Neurobiology of Disease 2015;75:151-8. doi:10.1016/j.nbd.2014.12.025.

[29] Kovac S, Domijan A-M, Walker MC, Abramov AY. Seizure activity results in calcium- and mitochondriaindependent ROS production via NADPH and xanthine oxidase activation. Cell Death Dis 2014;5:e1442. doi:10.1038/cddis.2014.390.

[30] Pauletti A, Terrone G, Shekh-Ahmad T, Salamone A, Ravizza T, Rizzi M, et al. Targeting oxidative stress improves disease outcomes in a rat model of acquired epilepsy. Brain 2019. doi:10.1093/brain/awz130.

[31] Kalita J, Misra UK, Singh LS, Tiwari A. Oxidative stress in status epilepticus: A clinical-radiological correlation. Brain Res 2019;1704:85-93. doi:10.1016/j.brainres.2018.09.038.

[32] Lehtinen MK, Tegelberg S, Schipper H, Su H, Zukor H, Manninen O, et al. Cystatin B Deficiency Sensitizes Neurons to Oxidative Stress in Progressive Myoclonus Epilepsy, EPM1. J Neurosci 2009;29:5910-5. doi:10.1523/JNEUROSCI.0682-09.2009.

[33] Turrens JF. Mitochondrial formation of reactive oxygen species. J Physiol (Lond) 2003;552:335-44. doi:10.1113/jphysiol.2003.049478.

[34] Malinska D, Kulawiak B, Kudin AP, Kovacs R, Huchzermeyer C, Kann O, et al. Complex III-dependent superoxide production of brain mitochondria contributes to seizure-related ROS formation. Biochim Biophys Acta 2010;1797:1163-70. doi:10.1016/j.bbabio.2010.03.001.

[35] Adam-Vizi V, Chinopoulos $\mathrm{C}$. Bioenergetics and the formation of mitochondrial reactive oxygen species. Trends in Pharmacological Sciences 2006;27:639-45. doi:10.1016/j.tips.2006.10.005.

[36] Kovac S, Domijan A-M, Walker MC, Abramov AY. Prolonged seizure activity impairs mitochondrial bioenergetics and induces cell death. J Cell Sci 2012;125:1796-806. doi:10.1242/jcs.099176.

[37] Williams S, Hamil N, Abramov AY, Walker MC, Kovac S. Status epilepticus results in persistent overproduction of reactive oxygen species, inhibition of which is neuroprotective. Neuroscience 2015;303:160-5. doi:10.1016/j.neuroscience.2015.07.005. 
[38] Babior BM, Kipnes RS, Curnutte JT. Biological defense mechanisms. The production by leukocytes of superoxide, a potential bactericidal agent. J Clin Invest 1973;52:741-4. doi:10.1172/JCl107236.

[39] Bedard K, Krause K-H. The NOX family of ROS-generating NADPH oxidases: physiology and pathophysiology. Physiol Rev 2007;87:245-313. doi:10.1152/physrev.00044.2005.

[40] Nayernia Z, Jaquet V, Krause K-H. New insights on NOX enzymes in the central nervous system. Antioxid Redox Signal 2014;20:2815-37. doi:10.1089/ars.2013.5703.

[41] Brennan AM, Suh SW, Won SJ, Narasimhan P, Kauppinen TM, Lee $\mathrm{H}$, et al. NADPH oxidase is the primary source of superoxide induced by NMDA receptor activation. Nat Neurosci 2009;12:857-63. doi:10.1038/nn.2334.

[42] Walker MC. Pathophysiology of status epilepticus. Neurosci Lett 2018;667:84-91. doi:10.1016/j.neulet.2016.12.044.

[43] Kim J-E, Ryu HJ, Kang T-C. Status epilepticus induces vasogenic edema via tumor necrosis factor- $\alpha$ / endothelin1-mediated two different pathways. PLoS ONE 2013;8:e74458. doi:10.1371/journal.pone.0074458.

[44] Huang W-Y, Lin S, Chen H-Y, Chen Y-P, Chen T-Y, Hsu K-S, et al. NADPH oxidases as potential pharmacological targets against increased seizure susceptibility after systemic inflammation. J Neuroinflammation 2018;15:140. doi:10.1186/s12974-018-1186-5.

[45] Lee SH, Choi BY, Kho AR, Jeong JH, Hong DK, Kang DH, et al. Inhibition of NADPH Oxidase Activation by Apocynin Rescues Seizure-Induced Reduction of Adult Hippocampal Neurogenesis. Int J Mol Sci 2018;19. doi:10.3390/ijms19103087.

[46] Baran H, Vass K, Lassmann H, Hornykiewicz O. The cyclooxygenase and lipoxygenase inhibitor BW755C protects rats against kainic acid-induced seizures and neurotoxicity. Brain Res 1994;646:201-6.

[47] Ueda Y, Yokoyama H, Niwa R, Konaka R, Ohya-Nishiguchi H, Kamada H. Generation of lipid radicals in the hippocampal extracellular space during kainic acid-induced seizures in rats. Epilepsy Res 1997;26:329-33.

[48] Acharya MM, Hattiangady B, Shetty AK. Progress in Neuroprotective Strategies for Preventing Epilepsy. Prog Neurobiol 2008;84:363-404. doi:10.1016/j.pneurobio.2007.10.010.

[49] Willmore LJ, Rubin JJ. Antiperoxidant pretreatment and iron-induced epileptiform discharges in the rat: EEG and histopathologic studies. Neurology 1981;31:63-9. doi:10.1212/wnl.31.1.63.

[50] Levy SL, Burnham WM, Hwang PA. An evaluation of the anticonvulsant effects of vitamin E. Epilepsy Res 1990;6:12-7.

[51] Levy SL, Burnham WM, Bishai A, Hwang PA. The anticonvulsant effects of vitamin E: a further evaluation. Can J Neurol Sci 1992;19:201-3.

[52] Ambrogini P, Minelli A, Galati C, Betti M, Lattanzi D, Ciffolilli S, et al. Post-seizure a-tocopherol treatment decreases neuroinflammation and neuronal degeneration induced by status epilepticus in rat hippocampus. Mol Neurobiol 2014;50:246-56. doi:10.1007/s12035-014-8648-2.

[53] Pansani AP, Cysneiros RM, Colugnati DB, Janjoppi L, Ferrari D, de Lima E, et al. Long-term monotherapy treatment with vitamin $E$ reduces oxidative stress, but not seizure frequency in rats submitted to the pilocarpine model of epilepsy. Epilepsy Behav 2018;88:301-7. doi:10.1016/j.yebeh.2018.09.027.

[54] Golechha M, Bhatia J, Ojha S, Arya DS. Hydroalcoholic extract of Emblica officinalis protects against kainic acidinduced status epilepticus in rats: evidence for an antioxidant, anti-inflammatory, and neuroprotective intervention. Pharm Biol 2011;49:1128-36. doi:10.3109/13880209.2011.571264.

[55] Golechha M, Chaudhry U, Bhatia J, Saluja D, Arya DS. Naringin protects against kainic acid-induced status epilepticus in rats: evidence for an antioxidant, anti-inflammatory and neuroprotective intervention. Biol Pharm Bull 2011;34:360-5.

[56] Huang H-L, Lin C-C, Jeng K-CG, Yao P-W, Chuang L-T, Kuo S-L, et al. Fresh green tea and gallic acid ameliorate oxidative stress in kainic acid-induced status epilepticus. J Agric Food Chem 2012;60:2328-36. doi:10.1021/jf203709q.

[57] Nomura S, Shimakawa S, Miyamoto R, Fukui M, Tamai H. 3-Methyl-1-phenyl-2-pyrazolin-5-one or Nacetylcysteine prevents hippocampal mossy fiber sprouting and rectifies subsequent convulsive susceptibility in a rat model of kainic acid-induced seizure ceased by pentobarbital. Brain Res 2014;1590:65-74. doi:10.1016/j.brainres.2014.05.017.

[58] Santos LFL, Freitas RLM, Xavier SML, Saldanha GB, Freitas RM. Neuroprotective actions of vitamin C related to decreased lipid peroxidation and increased catalase activity in adult rats after pilocarpine-induced seizures. Pharmacol Biochem Behav 2008;89:1-5. doi:10.1016/j.pbb.2007.10.007.

[59] Xavier SM, Barbosa CO, Barros DO, Silva RF, Oliveira AA, Freitas RM. Vitamin C antioxidant effects in hippocampus of adult Wistar rats after seizures and status epilepticus induced by pilocarpine. Neurosci Lett 2007;420:76-9. doi:10.1016/j.neulet.2007.04.056.

[60] Mishra V, Shuai B, Kodali M, Shetty GA, Hattiangady B, Rao X, et al. Resveratrol Treatment after Status Epilepticus Restrains Neurodegeneration and Abnormal Neurogenesis with Suppression of Oxidative Stress and Inflammation. Sci Rep 2015;5. doi:10.1038/srep17807.

[61] Wu Z, Xu Q, Zhang L, Kong D, Ma R, Wang L. Protective effect of resveratrol against kainate-induced temporal lobe epilepsy in rats. Neurochem Res 2009;34:1393-400. doi:10.1007/s11064-009-9920-0. 
[62] Petkova Z, Tchekalarova J, Pechlivanova D, Moyanova S, Kortenska L, Mitreva R, et al. Treatment with melatonin after status epilepticus attenuates seizure activity and neuronal damage but does not prevent the disturbance in diurnal rhythms and behavioral alterations in spontaneously hypertensive rats in kainate model of temporal lobe epilepsy. Epilepsy Behav 2014;31:198-208. doi:10.1016/j.yebeh.2013.12.013.

[63] Pearson-Smith JN, Liang L-P, Rowley SD, Day BJ, Patel M. Oxidative Stress Contributes to Status Epilepticus Associated Mortality. Neurochem Res 2017;42:2024-32. doi:10.1007/s11064-017-2273-1.

[64] Shekh-Ahmad T, Eckel R, Dayalan Naidu S, Higgins M, Yamamoto M, Dinkova-Kostova AT, et al. KEAP1 inhibition is neuroprotective and suppresses the development of epilepsy. Brain 2018;141:1390-403. doi:10.1093/brain/awy071.

[65] Itoh K, Wakabayashi N, Katoh Y, Ishii T, Igarashi K, Engel JD, et al. Keap1 represses nuclear activation of antioxidant responsive elements by Nrf2 through binding to the amino-terminal Neh2 domain. Genes Dev 1999;13:76-86. doi:10.1101/gad.13.1.76.

[66] Suzuki T, Yamamoto M. Stress-sensing mechanisms and the physiological roles of the Keap1-Nrf2 system during cellular stress. J Biol Chem 2017;292:16817-24. doi:10.1074/jbc.R117.800169.

[67] Dinkova-Kostova AT, Kostov RV, Canning P. Keap1, the cysteine-based mammalian intracellular sensor for electrophiles and oxidants. Arch Biochem Biophys 2017;617:84-93. doi:10.1016/j.abb.2016.08.005.

[68] Baird L, Dinkova-Kostova AT. The cytoprotective role of the Keap1-Nrf2 pathway. Arch Toxicol 2011;85:241-72. doi:10.1007/s00204-011-0674-5.

[69] Esteras N, Dinkova-Kostova AT, Abramov AY. Nrf2 activation in the treatment of neurodegenerative diseases: a focus on its role in mitochondrial bioenergetics and function. Biol Chem 2016;397:383-400. doi:10.1515/hsz-20150295.

[70] Holmström KM, Kostov RV, Dinkova-Kostova AT. The multifaceted role of Nrf2 in mitochondrial function. Curr Opin Toxicol 2016;1:80-91. doi:10.1016/j.cotox.2016.10.002.

[71] Mazzuferi M, Kumar G, van Eyll J, Danis B, Foerch P, Kaminski RM. Nrf2 defense pathway: Experimental evidence for its protective role in epilepsy. Ann Neurol 2013;74:560-8. doi:10.1002/ana.23940. 


\section{Figures Legends}

Figure 1: Putative mechanisms leading to neuronal death following calcium (and sodium) entry through NMDA receptors. NMDA receptor activation and calcium entry activate several enzymes NADPH oxidase (NOX) and nitric oxide synthase (NOS). Reactive oxygen species and nitric oxide form peroxynitrite, which is toxic to DNA, proteins and lipids. Calcium from the cytosol is taken up by mitochondria and excessive mitochondria load results in decreased ATP production, energy failure and failure to maintain cellular ionic gradients. Mitochondrial calcium accumulation and reactive oxygen species contribute to the formation of the mitochondrial permeability transition pore (mPTP), which further disrupts mitochondrial function, but also permit cytochrome $c$ into the cytosol where it can activate apoptotic pathways.

Figure 2: Nrf2 and KEAP1. Nrf2 is bound by KEAP1 which prevents translocations into the nucleus ans results in ubiquitination. ${ }^{* * *}$ represents endogenous reactive oxygen species or exogenous drug which bind to KEAP1 permitting Nrf2 translocation to the nucleus where it acts on the antioxidant response element to increase endogenous antioxidant defences.

Figure 3: KEAP1 inhibitor RTA-408 inhibits epileptogenesis and neuroprotects following status epilepticus. Status Epilepticus was induced with kainic acid and terminated with diazepam after 2 hours. The rats were then randomized to vehicle or RTA-408 once a day for three days and were then monitored for up to 4 months. Nine rats in each group were monitored for 12 weeks after first seizure and, 4 of those rats in each group were monitored for 15 weeks following first seizure. ${ }^{*} P<0.05$, ${ }^{* *} P<0.01$ and ${ }^{* * *} P<0.001$ by generalized log-linear mixed model followed by sequential Bonferroni post hoc test. 
Figure 1

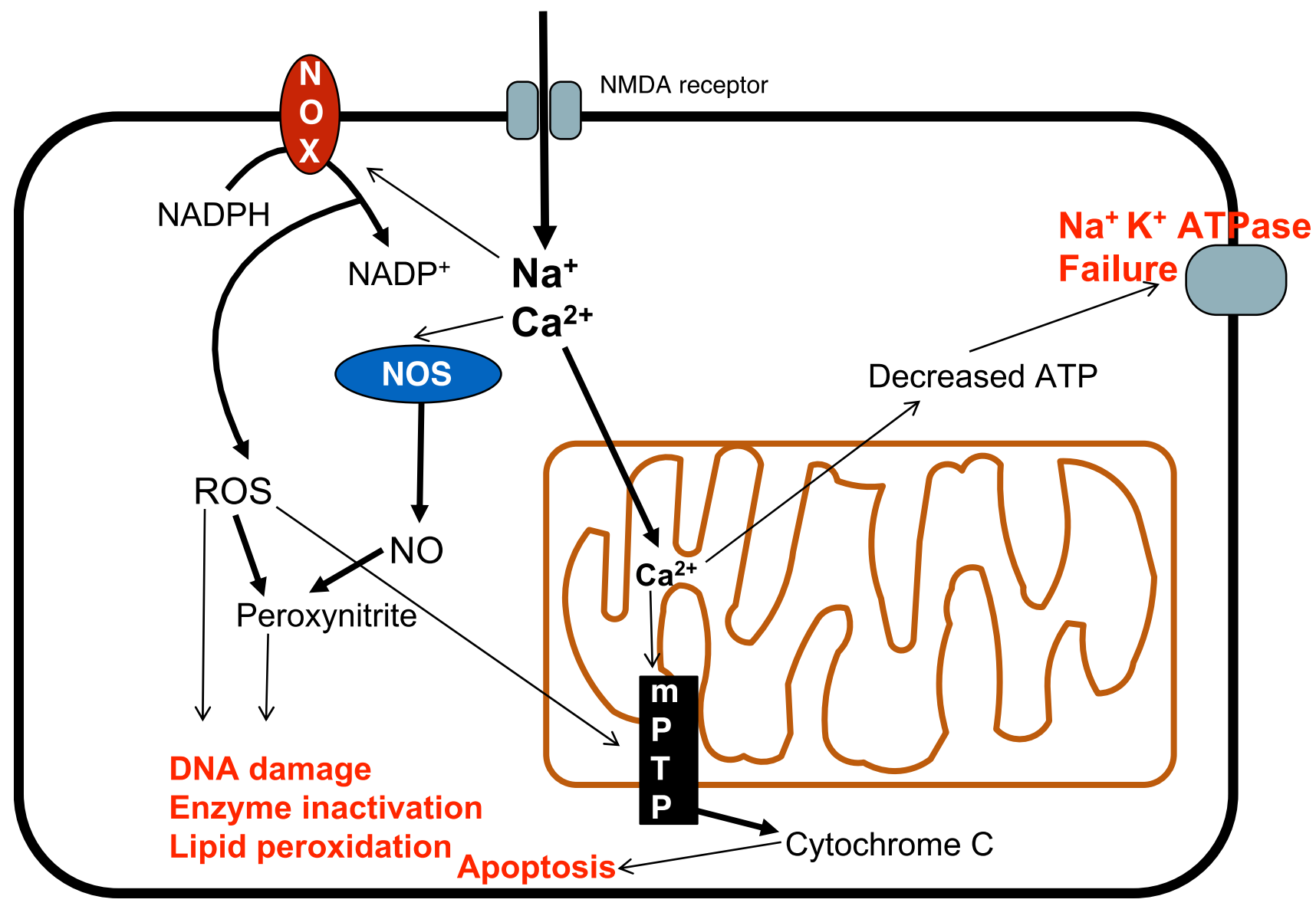


Figure 2

a



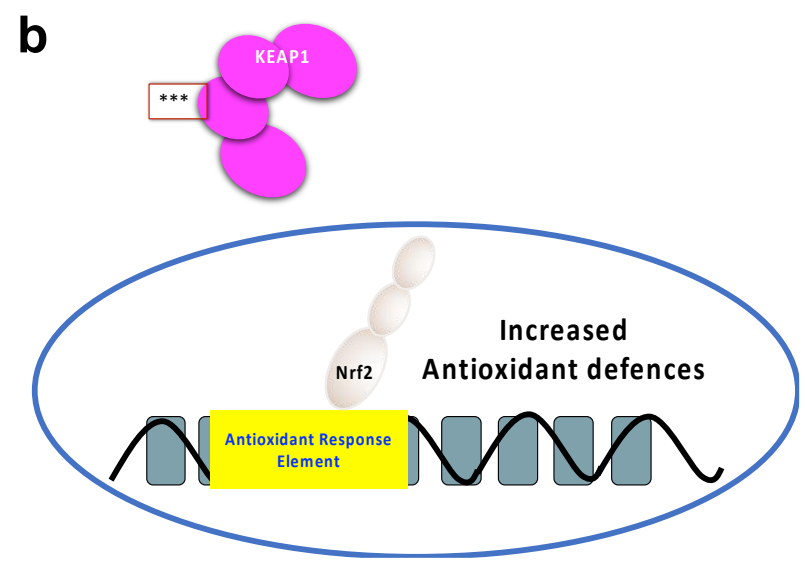


Figure 3
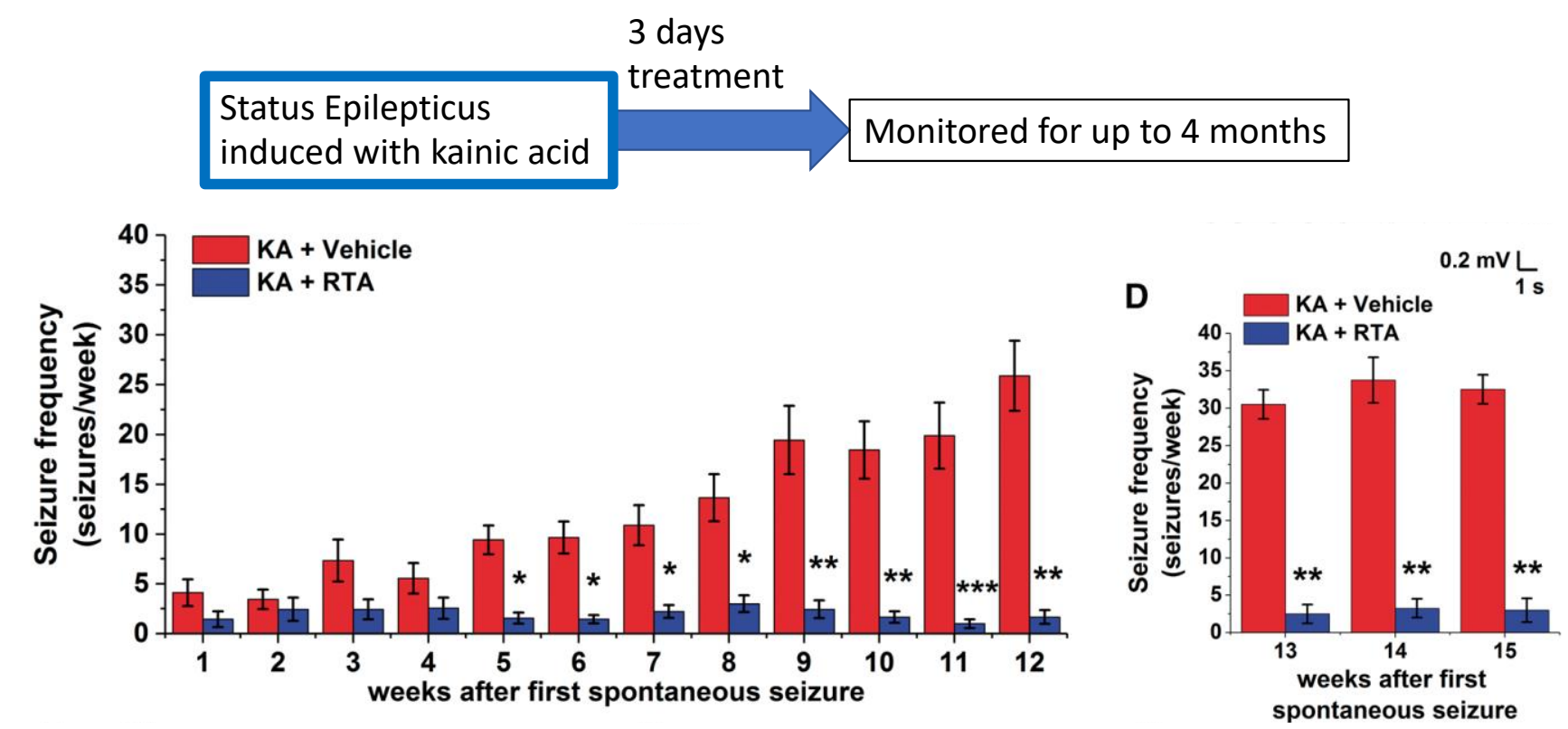